August 30, 2018

LBL-32856

UCB-PTH-92/35

\title{
A Candidate For The QCD String Based On Non-Backtracking Random Walk f
}

\author{
Korkut Bardakci \\ Department of Physics \\ University of California \\ and \\ Theoretical Physics Group \\ Physics Division \\ Lawrence Berkeley Laboratory \\ 1 Cyclotron Road \\ Berkeley, California 94720
}

\begin{abstract}
A new string theory is proposed as a candidate for the large $\mathrm{N}$ limit of QCD. In this theory, strings are constrained to be non-backtracking; a condition that is essential for the gauge invariance of the underlying field theory. This condition is implemented by first placing the theory on a lattice and then introducing fermionic variables on the world sheet. The naive continuum limit leads to a generalized Thirring model on the world sheet,and it is suggested that the application of the renormalization group should drive the coupling constants to a conformally invariant fixed point.
\end{abstract}

${ }^{*}$ This work was supported in part by the Director, Office of Energy Research, Office of High Energy and Nuclear Physics, Division of High Energy Physics of the U.S. Department of Energy under Contract DE-AC03-76SF00098 and in part by the National Science Foundation under grant PHY90-21139. 


\section{Disclaimer}

This document was prepared as an account of work sponsored by the United States Government. Neither the United States Government nor any agency thereof, nor The Regents of the University of California, nor any of their employees, makes any warranty, express or implied, or assumes any legal liability or responsibility for the accuracy, completeness, or usefulness of any information, apparatus, product, or process disclosed, or represents that its use would not infringe privately owned rights. Reference herein to any specific commercial products process, or service by its trade name, trademark, manufacturer, or otherwise, does not necessarily constitute or imply its endorsement, recommendation, or favoring by the United States Government or any agency thereof, or The Regents of the University of California. The views and opinions of authors expressed herein do not necessarily state or reflect those of the United States Government or any agency thereof of The Regents of the University of California and shall not be used for advertising or product endorsement purposes.

Lawrence Berkeley Laboratory is an equal opportunity employer. 


\section{Introduction}

It has been known for some time that in the large N limit,QCD without matter fields is equivalent to a free string theory(1).Despite considerable effort(2), this string theory is yet to be discovered.Recently,there has been renewed interest in this problem(3), as well as in other approaches to QCD at large $\mathrm{N}$ in general(4). Whatever it is, the QCD string cannot be the same as the standard Nambu-Polyakov string for at least the following reasons:

a)There are no zero mass particles(and of course no tachyon)in the QCD spectrum.

b)The Regge trajectories of QCD should be curved,as opposed to the straight trajectories of the standard string models. This is true of even two dimensional QCD coupled to matter(5).

One possible approach to this problem is to reformulate the dynamical equations of QCD in terms of gauge invariant quantities such as the Wilson loops. These equations have been derived for both the lattice(6) and the continuum(7) versions of the theory. Unfortunately,this approach has not thus far led to a simple string picture. One of the problems with the lattice appraoch is that the resulting string picture is extremely complicated(8).This is probably due to the simplicity of the starting point,which is the usual Wilson single plaquette interaction;it is conceivable that a more complicated interaction, which is in the same universality class and which therefore has the same continuum limit, may give rise to a simpler string. However,since it is not clear a priori how to determine the interaction that corresponds to a simple string picture on the lattice,it is tempting to bypass the lattice altogether and work directly in the continuum with Migdal-Makeenko equations(7).In this case,one encounters the problem of finding a suitable non-perturbative regulator to avoid divergences. So far,not much progress has been made along these lines.

In this paper,we bypass the difficult problem of showing directly the equivalence between QCD and the corresponding string theory;instead,we focus on the constraints satisfied by Wilson loops. One can think of the world sheet of the QCD string as being traced by a spacelike Wilson loop propagating in time,and in the Hamiltonian description of this process, the physical Hilbert space is generated by the collection of all spacelike Wilson loops. This forms an overcomplete 
set of states as a consequence of two kinds of constraints satisfied by the Wilson loops:

a)The Mandelstam identities(9). They follow from the fact that $\mathrm{N}$, the dimension of the color space, is finite.It is generally assumed that in the limit $N \rightarrow \infty$,there are no identities of this type.Since we are always in the large N limit in this paper,we will not consider them any further.

b)Every backtracking Wilson loop is equivalent to a non-backtracking one with the backtracking portion removed(see Fig.1).We shall argue in the next section that this equivalence is crucial for the existence of an underlying gauge theory;if this condition is not satisfied,Wilson loops cannot be constructed from a gauge connection. Conversely, if this condition holds, there always exists a connection valued in some (in general infinite dimensional) color space. This is the condition we focus on here.

In the standard bosonic string theory,one has an unrestricted sum over all possible world sheets, and therefore, taking a fixed time slice, all possible Wilson loops, backtracking as well as non-backtracking, are present. Since the backtracking loops are equivalent to the corresponding non-backtracking ones ,there is clearly some overcounting. At the minimum, a string theory based on QCD should avoid this overcounting and sum only on world sheets whose cross sections are non-backtracking loops. In this paper,we will study precisely such a string theory where the sum over the world sheets is suitably restricted.Because such a theory always admits a connection(see (b)above),it corresponds to some kind of gauge theory,and the question is,does it have the same interaction as QCD? In this paper,we shall leave this question open,and study this new string theory as an interesting theory in its own right. Since the underlying field theory is gauge invariant, it is a plausible candidate for the QCD string,hence the title of this paper. In a future publication(10),we hope to show that the version of the model where time is continuous and space is latticized corresponds to a gauge theory on a space lattice.However, the interaction is not the standard single plaquette interaction of Wilson, but instead it is a suitably weighed infinite sum over the traces of all possible Wilson loops. This sum has the standard QCD interaction as its naive continuum limit, and if we believe this naive limit, which we afterall do in the case of the single plaquette interaction,the equivalence to QCD follows. 
The technical problem that will occupy us for most of the rest of this paper is how to impose the condition that strings are non-backtracking. Since it is difficult to handle this condition directly in the continuum,we first start with a regular space-time lattice and only at the end take the continuum limit.As a warmup exercise,in section 2, we consider the simpler problem of non-backtracking random walk(Brownian motion), which involves summing over non-bactracking paths on the lattice. This problem can be solved in a number of different ways; the method which is easiest to generalize to sums over surfaces is the standard path integral approach.In order to implement the non-backtracking condition,we introduce additional fermionic variables,and we construct the action,making use of the transfer matrix method. The path integral must be supplemented by a boundary condition on the conserved fermion number to eliminate the unwanted backtracking paths.In section 3 ,this method is generalized to non-backtracking surfaces, which are defined as the direct product of two non-backtracking curves,and again a path integral formulation is obtained. The naive continuum limit of this model is discussed in section 4;the resulting two dimensional field theory that lives on the world sheet is a generalized Thirring model with the following properties:

a)It is scale invariant at the classical level.

b)It is Lorentz invariant in the target space.

c)It is Lorentz and parity invariant on the two dimensional world sheet as well. d)There is invariance under both vector and axial phase transformations of fermions,assuming absence of anomalies.As a result,there exists a chirally conserved fermion current on the world sheet.Just as in the case of random walk,suitable boundary conditions must be imposed on the conserved fermion current in order to eliminate the unwanted sectors of the model.

There are several coupling constants in world sheet field theory that are easily calculated in the naive continuum limit. Of course,this is not the final answer;these constants should be allowed to run under the renormalization group equations and reach a fixed point.We are unable to carry out this analysis,instead,we conjecture that there is a non-trivial fixed point and that the theory is conformally invariant at that point.Naturally,in searching for this fixed point, only those renormalization flows that preserve the invariances a) to d) should be considered.The main result of this paper is therefore the fol- 
lowing:The original problem of non-backtracking strings has been transformed into the problem of finding the conformal fixed point of a generalized Thirring model invariant under the symmetries listed above. We feel that this is a result of interest, although finding such a fixed point,and solving the resulting model,are difficult problems,and they will not be attempted here.So far,in problems of this type,solutions have been found only in very special cases(11), and hopefully,methods developed in the study of conformal theories should prove useful in tackling this problem in the future.More advanced problems such as the value of the central charge and the higher order corrections in $1 / N$ are also beyond the scope of this paper.

\section{Non-Backtracking Random Walk}

We start by fixing our notation and defining some terminology.Space-time (target space)is taken to be $\mathrm{D}$ dimensional;the value of $\mathrm{D}$ will not be fixed in this paper.The metric in the target space is flat and can be either Euclidean or Minkowski.We are interested in both open and closed strings;we identify the open string with the Wilson line integral

$$
U_{\Gamma}(x, y)=P\left\{\exp \int_{x}^{y} d x^{\prime} \cdot A\left(x^{\prime}\right)\right\}
$$

where $\Gamma$ is a path with endpoints $\mathrm{x}$ and $\mathrm{y}, \mathrm{A}$ is the connection valued in some (infinite dimensional) matrix space, and $\mathrm{P}$ denotes path ordering. $\mathrm{U}$ could be thought of as generating a line of flux connecting two external quarks at $\mathrm{x}$ and y along the path $\Gamma$.Closed strings correspond to

$$
W=\operatorname{Tr}\left\{U_{\Gamma}(x, x)\right\}
$$

for a closed path $\Gamma$.The equivalence between the U's associated with the backtracking path $\Gamma$ and the corresponding non-backtracking path $\Gamma^{\prime}$ pictured in Fig.1 follows from

$$
U_{\Gamma}(x, y)=U_{\Gamma^{-1}}^{-1}(y, x),
$$

where $\Gamma^{-1}$ is $\Gamma$ traversed in the opposite sense. As a result, the contributions to $U$ from the segments a and b in Fig.1 cancel. Of course, (2.2) follows immediately from (2.1a), but the converse is also true: If eq. (2.2) holds for arbitrary paths, and in addition, we also require the semi-group property

$$
U_{\Gamma^{(1,2)}}=U_{\Gamma^{(1)}} U_{\Gamma^{(2)}}
$$


for two arbitrary paths $\Gamma^{(1)}$ and $\Gamma^{(2)}$ that join at point $y$ and give $\Gamma^{(12)}$ (see Fig.2), then $U$ can be written in terms in terms of a connection $A$ as in (2.1). Therefore,eq's (2.2)and (2.3) guarantee the existence of an underlying gauge theory. In a random walk(or a string) problem,one has to deal with the paths directly, instead of the U's attached to them,and so it is convenient to define an abstract group generated by path multiplication (12). The group axioms are

$$
\Gamma(x, y) \Gamma^{-1}(y, x)=1
$$

and

$$
\Gamma^{(1,2)}(x, z)=\Gamma^{(1)}(x, y) \Gamma^{(2)}(y, z)
$$

where, again, multiplication of two paths consists of joining them beginning to end to form a new path(Fig.2). The U's can then be thought of as representations of the abstract group relations given by eqn's (2.4).It is easy see that, on the lattice, $(2.4 \mathrm{~b})$ is trivially satisfied if we build up the path(string)link by link; on the other, the cancellation condition (2.4a) is not automatic and remains to be implemented.

Before turning to the problem of non-bactracking random walk,as a preliminary exercise,we will briefly consider the simpler and extensively studied problem of random walk without constraints(13). This problem will be solved first by the operator and then by the path integral method. The reason for going through this simple exercise is that the methods used generalize easily to the real problem. We start with a regular lattice with spacing $\mathrm{d}$ in $\mathrm{D}$ dimensions, and label the lattice sites by the vector $\mathbf{x}$, whose components are given by $x_{a}$. Boldface letters will always denote vectors in the target space. Let the orthonormal set of states $\mid \mathbf{x}>$ be discrete eigenstates of $\mathbf{x}$ with the corresponding eigenvalue, and let $p_{a}=-i \frac{\partial}{\partial x_{a}}$ be the components of the conjugate momentum p. The operator $\exp \left(i d j p_{a}\right)$ shifts by one link in the positive "a" direction for $j=+1$ and by one link in the negative "a" direction for $j=-1$. We now consider a sum over all paths starting at $\mathbf{x}$ and ending at $\mathbf{x}^{\prime}$, each weighed by a Boltzmann factor $\exp (-\alpha l)$, where $\mathrm{l}=\mathrm{nd}$ is the length of the path. It is given by the following operator expression:

$$
G\left(\mathbf{x}, \mathbf{x}^{\prime}\right)=<\mathbf{x}^{\prime}\left|\left(1-2 e^{-\alpha d} \sum_{a} \cos \left(d p_{a}\right)\right)^{-1}\right| \mathbf{x}>
$$


We are also interested in the continuum limit $d \rightarrow 0$. This limit is taken by replacing the cosine by

$$
\sum_{a} \cos \left(d p_{a}\right) \approx D-\frac{1}{2} d^{2} \sum_{a} p_{a}^{2}
$$

and scaling $\mathrm{G}$ by

$$
\bar{G}=d^{2-D} e^{-\alpha d} G,
$$

while keeping $\kappa^{2}=\left(e^{\alpha d}-2 D\right) / d^{2}$ fixed as $d \rightarrow 0$. The Green's function $\mathrm{G}$ then satisfies the Helmholtz equation:

$$
\left(\kappa^{2}-\nabla_{x}\right) \bar{G}\left(\mathbf{x}, \mathbf{x}^{\prime}\right)=\delta^{D}\left(\mathbf{x}-\mathbf{x}^{\prime}\right),
$$

which is a well known result. Here, $\nabla=-\sum_{a} p_{a}^{2} \equiv-\mathbf{p}^{2}$.

The operator formulation of the random walk problem does not easily generalize to strings;instead, it is better to use the path integral approach. It is convenient to introduce link vectors $\mathbf{e}_{a}$ of unit length in the positive "a" direction and vectors $\mathbf{e}_{a, j}=j \mathbf{e}_{a}$ representing unit steps in + or - "a" direction,where $j= \pm 1$. The correlation function $\mathrm{G}$ is then given by

$$
G=\sum_{m=0}^{\infty} G^{(m)}
$$

where

$$
G^{(m)}=\int \prod_{m^{\prime}=1}^{m-1} d^{D} \mathbf{X}\left(m^{\prime}\right) \prod_{m^{\prime \prime}=1}^{m} d^{D} \mathbf{P}\left(m^{\prime \prime}\right) \exp \left(i I^{(m)}-(m-1) \alpha d\right),
$$

and,

$$
\exp \left(i I^{(m)}\right)=\sum_{\mathbf{e}} \exp \left(i \sum_{m^{\prime}=1}^{m} \mathbf{P}\left(m^{\prime}\right) \cdot\left(\mathbf{X}\left(m^{\prime}\right)-\mathbf{X}\left(m^{\prime}-1\right)-d \mathbf{e}_{a, j}\left(m^{\prime}\right)\right) .\right.
$$

In this equation,integration over $\mathbf{P}$ enforces the constraint that adjacent $\mathbf{X}^{\prime} s$ on the lattice differ by one of the link vectors $d \mathbf{e}_{a, j}$.It is also understood that the initial and final points of the path, $\mathbf{X}(0)$ and $\mathbf{X}(m)$, are fixed at $\mathbf{X}$ and $\mathbf{X}^{\prime}$.

In the continuum limit, the discrete parameter $\mathrm{m}$ is replaced by the continuous parameter $\tau=m d$. Again expanding to second order in $\mathrm{d}$ according to

$$
\sum_{a j} \exp \left(-i d \mathbf{e}_{a j} . \mathbf{P}\right) \approx \exp \left(\ln (2 D)-\frac{d^{2}}{2 D} \mathbf{P}^{2}\right)
$$


we have the continuum action

$$
i I=\int d \tau\left(2 i \mathbf{X}^{\prime} \cdot \mathbf{P}-\mathbf{P}^{2}-\kappa^{2}\right),
$$

which descibes a simple Gaussian model, in agreement with eq.(2.7). Here, $\kappa^{2}$ is a constant.

Next, we consider a non-backtracking random walk on the lattice. This is a Markov process, and it can be handled by slightly generalizing the operator approach we have used for the unrestricted random walk. Now we pick a point $\mathbf{X}$ on the path,with the previous step leading to it given by $\mathbf{e}_{a, j}$ and the following step by $\mathbf{e}_{b, k}$ (Fig.3). If the path is non-backtracking, $a=b, k=-j$ is not allowed. To take this into account, the state representing $\mathbf{X}$ should have the memory of the previous step. This is accomplished by attaching the indices "a" and " $\mathrm{j}$ " of the previous step to the state $\mid \mathbf{X}>$ by defining

$$
|\mathbf{X}, a, j>\equiv| \mathbf{X}>\otimes \mid a, j>
$$

where $\mid a, j>$ are orthonormal states. The two point correlation function (see eq.(2.5)) is then given by

$$
G\left(\mathbf{X}, a, j, \mathbf{X}^{\prime}, b, k\right)=<\mathbf{X}^{\prime}, b, k\left|\left(1-e^{-\alpha d} T\right)^{-1}\right| \mathbf{X}, a, j>,
$$

where the $2 \mathrm{D}$ by $2 \mathrm{D}$ matrix $\mathrm{T}$ is given by

$$
T_{b k, a j}=\exp \left(i d p_{b} k\right)\left(1-\delta_{a, b} \delta_{k,-j}\right),
$$

and $p_{a}=-i \frac{\partial}{\partial x_{a}}$ as before. It is easy to check that $\mathrm{T}$ does exactly what it is supposed to do; it moves $\mathbf{X}$ by one step in all directions except the backtracking one.

To gain some insight into what is going on, it is useful to diagonalize $\mathrm{T}$ for fixed p.Defining $\omega=2 \sum_{a} \cos \left(d p_{a}\right)$, the four distinct eigenvalues are given by

$$
\begin{aligned}
& \lambda^{(1)}=\frac{\omega}{2}+\frac{1}{2}\left(\omega^{2}-8 D+4\right)^{1 / 2}, \\
& \lambda^{(2)}=\frac{\omega}{2}-\frac{1}{2}\left(\omega^{2}-8 D+4\right)^{1 / 2}, \\
& \lambda^{(3)}=+1, \\
& \lambda^{(4)}=-1 .
\end{aligned}
$$


The corresponding eigenvectors can be written in the following form:

$$
\left|i>=\sum_{a j} \alpha_{a, j}^{(i)}\right| a, j>,
$$

where the $\alpha$ 's are constants, and the index $i=1,2,3,4$ labels the eigenvectors corresponding to the eigenvalues $\lambda^{(1,2,3,4)}$. The equations satisfied by the $\alpha$ 's are

$$
\begin{aligned}
& \alpha_{a, j}^{(1,2)}=1-\lambda^{(1,2)} \exp \left(i d j p_{a}\right), \\
& \sum_{a j} \alpha_{a, j}^{(3,4)}=0 \\
& \alpha_{a,-1}^{(3)}=-\exp \left(-i d p_{a}\right) \alpha_{a, 1}^{(3)}, \\
& \alpha_{a,-1}^{(4)}=\exp \left(-i d p_{a}\right) \alpha_{a, 1}^{(4)} .
\end{aligned}
$$

Since $\mathrm{T}$ is not hermitian,the eigenvectors are not orthogonal. This apparent lack of symmetry between the past and the future is due to the way we chose to label the states, and it does not have any physical consequence. For example,physical quantities like the eigenvalues are real. We also note that the eigenvectors corresponding to $i=1,2$ are non-degenerate, whereas the eigenvectors corresponding to $i=3,4$ are each $D-1$ fold degenerate. It is useful to expand $\lambda^{(1,2)}$ to second order in $\mathrm{d}$ in anticipation of the continuum limit:

$$
\begin{aligned}
& \lambda^{(1)} \cong 2 D-1-\frac{2 D-1}{2 D-2} d^{2} \mathbf{p}^{2}, \\
& \lambda^{(2)} \cong 1+\frac{1}{2 D-2} d^{2} \mathbf{p}^{2} .
\end{aligned}
$$

In the limit $d \rightarrow 0$, the eigenvector corresponding to the eigenvalue $\lambda^{(1)}$ dominates over the rest; and therefore, in the continuum limit, we could drop all the eigenvectors except the first one.However, we shall see later that this is no longer true in the case of strings; accordingly, we postpone the discussion of the continuum limit to the next section. In passing, we note that the zeroth order term in the expansion of $\lambda^{(1)}$ is reduced from $2 \mathrm{D}$ to $2 D-1$ due to the fact that the step in the backtracking direction is no longer allowed.

Let us now recast eq.(2.11) into the path integral language, with the view of generalizing it to strings later. In the unconstrained random walk problem, only $\mathbf{X}$ and $\mathbf{P}$ were needed as variables of integration.However, since we now 
have discrete indices a and $\mathrm{j}$ to deal with, additional variables of integration have to be introduced. These variables could be either bosonic or fermionic, and in one and two dimensional spaces, they are known to be equivalent.(Here we are referring to the dimensions of the world line and the world sheet). We find that fermionic variables have several advantages over the bosonic ones; for one thing, the formulas look much simpler, and also finitely indexed fermions naturally span a finite dimensional space due to the exclusion principle. Let us therefore introduce fermionic c-number variables $\bar{\eta}_{a, j}(m)$ and $\eta_{a, j}(m)$ to keep track of the indices a and $\mathrm{j}$. Then the $I^{(m)}$ of eq.(2.8b) can in the non-backtracking case be written as

$$
\begin{aligned}
i I^{(m)} & =\sum_{m^{\prime}=1}^{m-1}\left(i \mathbf{P}\left(m^{\prime}\right) \cdot\left(\mathbf{X}\left(m^{\prime}\right)-\mathbf{X}\left(m^{\prime}-1\right)\right)\right. \\
& +\frac{i}{2} \sum_{a j}\left(\bar{\eta}_{a, j}\left(m^{\prime}\right) \eta_{a, j}\left(m^{\prime}+1\right)-\bar{\eta}_{a, j}\left(m^{\prime}+1\right) \eta_{a, j}\left(m^{\prime}\right)\right) \\
& \left.+\sum_{a j, b k} \bar{\eta}_{b, k}\left(m^{\prime}\right) M_{b k, a j}\left(m^{\prime}\right) \eta_{a, j}\left(m^{\prime}\right)\right) .
\end{aligned}
$$

To establish connection with the operator formulation of the same problem and to find $\mathrm{M}$, the transfer matrix for the $\eta^{\prime} s$ should be identified with the matrix $\mathrm{T}$ of (2.11b). Remembering that $T_{b k, a j}(m)$ maps the state $\mid a, j>$ at step m into the state $\mid b, k>$ at step $\mathrm{m}+1$, we must have

$$
\eta(m+1)=T(m) \eta(m),
$$

where we have used the matrix notation. Comparing this with the equations of motion for $\eta$ that follow from the lagrangian of eq.(2.15),

$$
\frac{i}{2}(\eta(m+1)-\eta(m-1))+M(m) \eta(m)=0,
$$

gives us the relation between $\mathrm{M}$ and $\mathrm{T}$ :

$$
\frac{i}{2}\left(T(m)-T^{-1}(m-1)\right)+M(m)=0 .
$$

Parenthetically, the expression for $\mathrm{T}$ given in $(2.11 \mathrm{~b})$ should be slightly modified: The operator $p_{b}$ should be replaced by the integration variable $P_{b}(m)$. This is why $\mathrm{T}$ acquires an apparent $\mathrm{m}$ dependence. 
The action of eq.(2.15), with $\mathrm{M}$ given by (2.16c), is still not completely equivalent to the operator approach; it has to be supplemented by an important boundary condition. The transfer matrix $\mathrm{T}(\mathrm{m})$ maps a single fermion at step $\mathrm{m}$, represented by $\eta(m)$,into again a single fermion at step $m+1$. On the other hand,once we have a functional integral, the resulting field theory will have all the states containing arbitrary number of fermions, up to the maximum allowed by fermi statistics. These extra states are unwanted; for example,in the zero fermion sector(vacuum), the T matrix is trivial (unity), and this sector therefore describes the unconstrained random walk. To eliminate these unwanted sectors, we note that the fermion number is conserved, and sectors with different fermion numbers do not mix. We can therefore impose the boundary condition that the initial state has fermion number one; this condition will be preserved by the dynamics. This initial condition is the extra ingredient that supplements the action given by eq.(2.15).

\section{Non-Backtracking Strings}

In this section, we will try to extend the results obtained so far to nonbacktracking strings. We have the same D dimensional lattice of the last section, and as a preliminary exercise, we first start with the ordinary unconstrained sum over surfaces traced by a string(string world sheet). At this point, we have to define what we mean by a surface. It is easy to think of several reasonable definitions; most of these, possibly even all of them, will have the same continuum limit.Here we choose a simple definition: Consider the coordinate variable $X_{a}(m, n)$, parametrized by two integers $\mathrm{m}$ and $\mathrm{n}$. The surface is defined by requiring that two adjacent points on it are connected by a link vector, hence the following equations must hold for some $a, j, a^{\prime}, j^{\prime}$ :

$$
\begin{aligned}
& \mathbf{X}(m, n)-\mathbf{X}(m-1, n)=d \mathbf{e}_{a, j}^{(1)}(m, n), \\
& \mathbf{X}(m, n)-\mathbf{X}(m, n-1)=d \mathbf{e}_{a^{\prime}, j^{\prime}}^{(2)}(m, n),
\end{aligned}
$$

where the $\mathbf{e}^{\prime} s$ are the same unit vectors defined in the last section, and superscripts (1) and (2) are attached to distinguish between them. These constraints can be implemented by introducing the lagrange multipliers $\mathbf{P}^{(1)}$ and $\mathbf{P}^{(2)}$, and 
the partition function $\mathrm{Z}$ as a sum over surfaces is then given by

$$
\begin{aligned}
Z= & \int \prod_{m n} d^{D} \mathbf{X}(m, n) d^{D} \mathbf{P}^{(1)}(m, n) d^{D} \mathbf{P}^{(2)}(m, n) \\
& \sum_{a j a^{\prime} j^{\prime}} \exp \left(i \sum _ { m n } \left(\mathbf{P}^{(1)}(m, n) \cdot\left(\mathbf{X}(m, n)-\mathbf{X}(m-1, n)-d \mathbf{e}_{a, j}^{(1)}(m, n)\right)\right.\right. \\
& \left.+\mathbf{P}^{(2)}(m, n) \cdot\left(\mathbf{X}(m, n)-\mathbf{X}(m, n-1)-d \mathbf{e}_{a^{\prime}, j^{\prime}}^{(2)}(m, n)\right)\right) .
\end{aligned}
$$

Here, for simplicity, we have not introduced a term propotional to the area (Nambu term) in the exponential, since, as we shall see, such a term is automatically generated in any case. To arrive at a correlation function anologous to $G$ of the random walk problem(see eq.(2.8)), again one has to fix the initial and the final $\mathbf{X}^{\prime} s$ and sum over the number of steps it takes to get from one to the other. Let us now consider the naive(formal) continuum limit of this equation. The action in this limit is gotten by expanding in powers of $\mathrm{d}$, and by dropping terms that vanish in the limit $d \rightarrow 0$. It is of course necessary to scale the field variables correctly to end up with the correct continuum kinetic energy terms. Defining the coordinates on the world sheet by

$$
\sigma_{1}=m d, \sigma_{2}=n d,
$$

and scaling by

$$
\begin{aligned}
\mathbf{P}^{(1,2)} & \rightarrow(2 D)^{1 / 2} \mathbf{P}^{(1,2)} \\
\mathbf{X} & \rightarrow(2 / D)^{1 / 2} d \mathbf{X}
\end{aligned}
$$

we arrive at the result,

$$
i I \rightarrow \int d^{2} \sigma \sum_{l=1,2}\left(2 i \mathbf{P}^{(l)}(\sigma) \cdot \frac{\partial \mathbf{X}(\sigma)}{\partial \sigma_{l}}-\left(\mathbf{P}^{(l)}(\sigma)\right)^{2}\right)
$$

A few comments on this result are in order:

a)Integrating over $\mathbf{P}$ reproduces the action for the standard bosonic string, gauge fixed in the conformal gauge.However, the gauge fixing terms(ghosts) are missing. This presumably comes about because the naive continuum limit misses subtle quantum effects. It is easily fixed by the standard procedure of introducing the world sheet metric, casting (3.4) into reparametrization invariant form, and 
finally fixing the gauge(14).

b) The continuum result is rotationally invariant on the world sheet. This is of course essential for being able to generalize it to a reparametrization invariant form; since we have a constant background metric on the world sheet, the only remnant of reparametrization invariance is a global rotation invariance, and this should remain unbroken. There is even a discrete residue of this symmetry in the case of the lattice: Eq.(3.2) is invariant under $m \leftrightarrow n, 1 \leftrightarrow 2$. When we introduce fermionic variables later,this symmetry will generalize to a symmetry between the right and left chiral sectors of the model.

c)It was pointed out earlier that the definition of a world sheet surface was nonunique. In fact,the concept of surface is not central in our approach. All we require is that, upon identifying $\mathrm{n}$ with a discrete time variable,the coordinate vector $\mathbf{X}(m, n)$ should trace all the acceptable Wilson lines(loops) for a fixed $\mathrm{n}$ and variable $\mathrm{m}$. On account of the discrete symmetry discussed above,this should be true also for fixed $m$ and variable $n$. Therefore, in our approach, the world sheet can be thought of as a direct product of two sets of Wilson lines, traced by $\mathbf{X}(m, n)$ for either fixed $\mathrm{m}$ and variable $\mathrm{n}$, or for fixed $\mathrm{n}$ and variable $\mathrm{m}$ respectively.

As it stands, eq.(3.2) does not generate a satisfactory string theory, since backtracking Wilson loops are present in the sum. To eliminate them, we proceed as in the random walk problem discussed in section 2 . To the first set of Wilson lines, we attach the fermionic variables $\eta_{a, j}^{(1)}(m, n)$, and to the second set the variables $\eta_{a, j}^{(2)}(m, n)$; these variables "guide" the path so that it is nonbacktracking. For a single path, the transfer matrix $\mathrm{T}$ for the $\eta^{\prime} s$ is given by eq. (2.11b). In the present case, we have two transfer matrices $T^{(1,2)}$, each given by equation $(2.11 \mathrm{~b})$, with $p_{b}$ replaced by $P_{b}^{(1)}(m, n)$ and $P_{b}^{(2)}(m, n)$ respectively. The partition function is therefore given by

$$
\begin{aligned}
Z= & \int \mathcal{D} X \mathcal{D} P^{(1)} \mathcal{D} P^{(2)} \mathcal{D} \eta^{(1)} \mathcal{D} \eta^{(2)} \mathcal{D} \bar{\eta}^{(1)} \mathcal{D} \bar{\eta}^{(2)} \exp (i I), \\
& \text { where } \\
I= & I_{1}+I_{2}+I_{3}, \\
& \text { and, }
\end{aligned}
$$




$$
\begin{aligned}
I_{1}= & \sum_{m n}\left(\mathbf{P}^{(1)}(m, n) \cdot(\mathbf{X}(m, n)-\mathbf{X}(m-1, n))+\mathbf{P}^{(2)}(m n) \cdot(\mathbf{X}(m, n)-\mathbf{X}(m, n-1))\right) \\
I_{2}= & \frac{i}{2} \sum_{m n}\left(\bar{\eta}^{(1)}(m, n) \eta^{(1)}(m+1, n)-\bar{\eta}^{(1)}(m+1, n) \eta^{(1)}(m, n)\right. \\
& \left.+\bar{\eta}^{(2)}(m, n) \eta^{(2)}(m, n+1)-\bar{\eta}^{(2)}(m, n+1) \eta^{(2)}(m, n)\right) \\
I_{3}= & \sum_{m n} \sum_{l=1,2} \bar{\eta}^{(l)}(m, n) M^{(l)}(m, n) \eta^{(l)}(m, n)
\end{aligned}
$$

In this equation, we have again adopted the matrix notation and suppressed the indices a and $\mathrm{j}$ attached to the $\eta^{\prime} s$.From the definition of $\mathrm{T}$ and the equation of motion for $\eta$, the following relation between $\mathrm{T}$ and $\mathrm{M}$ follows(see eq.(2.16b)):

$$
\begin{aligned}
\frac{i}{2}\left(T^{(1)}(m, n)-\left(T^{(1)}(m-1, n)\right)^{-1}\right)+M^{(1)}(m, n) & =0 \\
\frac{i}{2}\left(T^{(2)}(m, n)-\left(T^{(2)}(m, n-1)\right)^{-1}\right)+M^{2}(m, n) & =0
\end{aligned}
$$

where $\mathrm{T}$ is given by

$$
T_{b k, a j}^{(1,2)}(m, n)=\exp \left(i d k P_{b}^{(1,2)}(m, n)\right)\left(1-\delta_{a, b} \delta_{k,-j}\right)
$$

Eqn's (3.5) and (3.6) describe the dynamics of non-bactracking paths in the functional approach. As explained at the end of section 2, they have to be supplemented by boundary conditions on the fermion number. These boundary conditions must ensure that one and only one fermion is attached to each of the fixed $m$ and the fixed $n$ trajectories. In the case of random walk, the existence of a conserved fermion number was crucial in preserving the boundary condition along the trajectory. Here, in contrast, we have two sets of conserved fermion number densities $N^{(1)}(m, n)$ and $N^{(2)}(m, n)$, where the first denotes the number of fermions propagating along the link connecting $(m, n)$ to $(m+1, n)$, and the second those propagating along the link $(m, n)$ to $(m, n+1)$. The conservation laws, which follow from (3.5), are

$$
N^{(1)}(m+1, n)=N^{(1)}(m, n), N^{(2)}(m, n+1)=N^{(2)}(m, n) .
$$

The following boundary conditions are then imposed: For a fixed initial value $m_{i}$ of $\mathrm{m}$, we require that

$$
N^{(1)}\left(m_{i}, n\right)=1
$$


and, for a fixed initial value $n_{i}$ of $\mathrm{n}$, the condition is

$$
N^{(2)}\left(m, n_{i}\right)=1
$$

These conditions are preserved along each trajectory by virtue of the conservation laws, and they eliminate unwanted sectors, including the sector $N^{(1)}=$ $N^{(2)}=0$, which corresponds to the standard bosonic string of eq.(3.2).

Is the string theory defined by eq.(3.5) the QCD string? In general, we cannot answer this question for a string that lives on a lattice. However, we expect to show in a future publication(10) that in the limit of continuous time, still keeping space latticized, the string theory we have constructed is equivalent to a gauge theory on a space lattice. This theory differs from the standard hamiltonian lattice QCD in having non-minimal interaction terms, although the formal continuum limit is the same. It is therefore reasonable to hope that the continuous space-time limit of our model, which we will study in the next section, is in fact the large $\mathrm{N}$ limit of QCD.

\section{The Continuum Limit}

In this section, we shall study the naive(or formal) continuum limit of eq.(3.5). The world sheet coordinates $\sigma_{1,2}$ are defined and bosonic variables $\mathbf{X}$ and $\mathbf{P}$ are scaled as before (eqs.(3.3a,b)), whereas the fermionic variables are scaled by

$$
\eta^{(1,2)}(m, n) \rightarrow d^{1 / 2} \eta^{1,2}\left(\sigma_{1}, \sigma_{2}\right) .
$$

The continuum limits of $I_{1,2}$ are straightforward to compute:

$$
\begin{aligned}
& I_{1} \rightarrow \int d^{2} \sigma \sum_{l=1,2} \mathbf{P}^{(l)} \cdot \frac{\partial \mathbf{X}}{\partial \sigma_{l}} \\
& I_{2} \rightarrow i \int d^{2} \sigma \sum_{l=1,2} \bar{\eta}^{(l)} \frac{\partial \eta^{(l)}}{\partial \sigma_{l}} .
\end{aligned}
$$

Finding the limit of $I_{3}$ involves little a bit more work. It is convenient to first diagonalize T (see eqs.(2.12) and (2.13)) before taking the $d \rightarrow 0$ limit, and then compute $\mathrm{M}$ through eq.(2.16c). In this calculation, only terms up to first order in $d$ are needed, since higher order terms do not contribute in this limit. To first 
order, the eigenvalues $\Lambda_{i}$ of $\mathrm{M}$ are

$$
\begin{aligned}
& \Lambda_{1} \cong \frac{i}{2}\left(\frac{1}{2 D-1}-2 D+1\right), \\
& \Lambda_{2} \cong 0 \\
& \Lambda_{3}=0 \\
& \Lambda_{4}=0
\end{aligned}
$$

and the right and the left eigenvectors $\mid i>$ and $<i \mid$ are given as a sum over states $\mid a, j>$ and $<a, j \mid$ (see eq.(2.10) for the definition):

$$
\begin{aligned}
& \left|1>\cong(2 D)^{-1 / 2} \sum_{a j}\left(1+i d \frac{2 D-1}{2 D-2} P_{a} j\right)\right| a, j> \\
& <1\left|\cong(2 D)^{-1 / 2} \sum_{a j}\left(1+\frac{i d}{2 D-2} P_{a} j\right)<a, j\right| .
\end{aligned}
$$

The states $|2>| 3>$, and $\mid 4>$ are not needed, since, the corresponding eigenvalues being 0 , they do not appear in the expansion of $\mathrm{M}$. Notice that as $\mathrm{T}$ and $\mathrm{M}$ are not hermitian, the left eigenvector $<1 \mid$ is not the conjugate of the right eigenvector $\mid 1>$. Also, the terms $\mathrm{T}$ and $T^{-1}$ in (3.6a) have different arguments, and this causes some confusion about what the argument of $P_{a}$ should be in eq.(4.3b). However, replacing $m-1$ by $\mathrm{m}$, or $n-1$ by $n$ changes the final result only by terms of order $d^{2}$ and therefore it is permissible. The matrix M, again to first order in $\mathrm{d}$, is given by

$$
M_{b k, a j}^{(1,2)}=\frac{i \Lambda_{1}}{2 D}\left(1+i d \frac{2 D-1}{2 D-2} P_{b}^{(1,2)} k+\frac{i d}{2 D-2} P_{a}^{(1,2)} j\right) .
$$

Substituting eq.(4.4) in (3.5) then gives the continuum limit of $I_{3}$. This term can be simplified substantially by defining a new set of $\eta^{\prime} s$ by

$$
\begin{aligned}
s^{(l)} & =(2 D)^{-1 / 2} \sum_{a j} \eta_{a, j}^{(l)}, \\
v_{a}^{(l)} & =2^{-1 / 2} \sum_{j} j \eta_{a, j}^{(l)}, \\
w_{c}^{(l)} & =\sum_{a j} f_{c, a} \eta_{a, j}^{(l)}
\end{aligned}
$$


and a new set of $\bar{\eta}^{\prime} s$ through the same equations. Here, $l=1,2$ as before, and the index c takes on $2 D-2$ values. The real constants $f_{c, a}$ satisfy the conditions

$$
\begin{aligned}
\sum_{a} f_{c, a} f_{c^{\prime}, a} & =\delta_{c, c^{\prime}}, \\
\sum_{a} f_{c, a} & =0 .
\end{aligned}
$$

Eqn.'s $(4.5 \mathrm{a}, \mathrm{b})$ are a set of orthogonal transformations to a new set of variables $s^{(l)}, v_{a}^{(l)}$ and $w_{c}^{(l)}$, which diagonalize $\mathrm{M}$, and which give the following simple result for $I_{3}$ :

$$
I_{3} \rightarrow \Lambda_{1} \int d^{2} \sigma \sum_{l}\left(\frac{i}{d} \bar{s}^{(l)} s^{(l)}-\frac{1}{D^{1 / 2}(2 D-2)} \sum_{a}\left(\bar{s}^{(l)} v_{a}^{(l)}+(2 D-1) \bar{v}_{a}^{(l)} s^{(l)}\right) P_{a}^{(l)}\right) .
$$

Now that we have the continuum limit, let us discuss some of its features:

a)The theory is rotation invariant in the $\mathrm{D}$ dimensional target space, provided that we assign the following transformation laws to the fields: $P_{a}^{(1,2)}, v_{a}^{(1,2)}$ and $\bar{v}_{a}^{(1,2)}$ are $\mathrm{D}$ vectors, and the rest, $s^{1,2}$ and $w_{c}^{(1,2)}$, are all scalars. This assignment follows uniquely from the fact that $X_{a}$ is a $\mathrm{D}$ vector. If so desired, one can then introduce the Minkowsky metric in the target space and end up with a Lorentz invariant theory.

b) The fields $w_{c}^{(1,2)}$ decouple from the rest of the theory and become free fields. Of course, when a metric is introduced on the world sheet to restore reparametrization invariance, they will couple to this metric and will contribute to the value of the central charge and to the calculation of higher order terms in $1 / N$. Since we will not study these more subtle issues in this paper, from now on these fields will be dropped.

c) Since the background metric is flat, depending on its signature, we expect to have either Lorentz or rotation symmetry on the world sheet. To see how this works, we first consider the kinetic energy terms for the field $\eta$ in eq.(4.2). From the structure of these terms, it is clear that $\eta^{(1)}$ and $\eta^{(2)}$ have to be identified with the the two chiral (left and right) components of a world sheet spinor. Accordingly, we define the following two component spinors:

$$
\begin{aligned}
& \eta_{a, j} \equiv\left(\begin{array}{c}
\eta_{a, j}^{(1)} \\
\eta_{a, j}^{(2)}
\end{array}\right), \\
& \bar{\eta}_{a, j} \equiv\left(\bar{\eta}_{a, j}^{(2)}, \bar{\eta}_{a, j}^{(1)}\right),
\end{aligned}
$$


with a similar definition for s and $v_{a} . I_{2}$ then becomes the standard Dirac kinetic energy term:

$$
I_{2}=i \int d^{2} \tau \sum_{a j} \bar{\eta}_{a, j} \gamma^{\mu} \frac{\partial \eta_{a, j}}{\partial \tau^{\mu}}
$$

where $\mu=0,1$, and,

$$
\begin{aligned}
& \gamma^{0}=\sigma_{x}, \gamma^{1}=i \sigma_{y}, \\
& \tau^{0}=\sigma_{1}+\sigma_{2}, \tau^{1}=\sigma_{1}-\sigma_{2} .
\end{aligned}
$$

In this equation, $\sigma_{x, y}$ are Pauli matrices, not to be confused with $\sigma_{1,2}$, the world sheet coordinates. From the structure of the Dirac equation, it is clear that $\sigma_{1,2}$ are the lightcone coordinates, in contrast to $\tau^{0,1}$, the Cartesian coordinates. It then follows that the world sheet metric is Minkowskian, which we have already anticipated by writing the path integral (3.5) in the Feynman form, with a factor "i" in front of the action I in the exponential. We also need the covariant two-vecor components $P_{a}^{\mu}$ of momentum in terms of the lightcone components $P_{a}^{1,2}$ :

$$
P_{a}^{0}=P_{a}^{(1)}+P_{a}^{(2)}, P_{a}^{1}=P_{a}^{(1)}-P_{a}^{(2)} .
$$

With these assignments of transformation laws for the fields, the second and the third terms on the right hand side of eq.(4.6) can be written in world sheet Lorentz invariant form:

$$
\begin{aligned}
\sum_{a l} \bar{v}_{a}^{(l)} s^{(l)} P_{a}^{(l)} & =\frac{1}{2} \sum_{a, \mu} \bar{v}_{a} \gamma^{\mu} s P_{a, \mu}, \\
\sum_{a l} \bar{s}^{(l)} v_{a}^{(l)} P_{a}^{(l)} & =\frac{1}{2} \sum_{a, \mu} \bar{s} \gamma^{\mu} v_{a} P_{a, \mu} .
\end{aligned}
$$

However, the first term on the right hand side of (4.6), proportional to,

$$
S \equiv \frac{1}{d} \sum_{l} \bar{s}^{(l)} s^{(l)}
$$

is not world sheet Lorentz invariant as it stands. We will return to this problem later.

d)All of the terms but one in the action have dimensionless coupling constants and therefore, they are classically scale invariant. The only exception is again 
S, the Lorentz non-invariant term discussed above.

e) The action is parity invariant (left-right symmetric) on the world sheet.

We will now show that the troublesome term $\mathrm{S}$ can be cast into a form that is both scale and Lorentz invariant. To be able to do this, it is important to realize that the form of the action is not unique; one can multiply any one of its terms by one of the number density operators constrained to be unity(eqs.(3.8a,b)). Expressed in terms of the fermionic variables on the lattice, these constraints can be written as

$$
\begin{aligned}
1 & =N^{(l)}(m, n) \rightarrow \sum_{a j} \bar{\eta}_{a, j}^{(l)}(m, n) \eta_{a j}^{(l)}(m, n) \\
& =\bar{s}^{(l)} s^{(l)}+\sum_{a} \bar{v}_{a}^{(l)} v_{a}^{(l)}+\sum_{c} \bar{w}_{c}^{(l)} w_{c}^{(l)} .
\end{aligned}
$$

In the continuum limit, this equation becomes

$$
d\left(\bar{s}^{(l)}(\tau) s^{(l)}(\tau)+\sum_{a} \bar{v}_{a}^{(l)}(\tau) v^{(l)}(\tau)\right) \cong 1,
$$

where, as explained earlier, the term involving w's was dropped. The factor of $\mathrm{d}$ comes from the scaling of the fields in passing to the continuum limit. The term $\mathrm{S}$, which is quadratic in field variables, can now be converted into a quartic term by multiplying it by a suitable combination of terms that appear on the right hand side of (4.10b):

$$
\begin{aligned}
S & =\frac{1}{d}\left(N^{(1)} \bar{s}^{(2)} s^{(2)}+N^{(2)} \bar{s}^{(1)} s^{(1)}\right) \\
& \rightarrow \frac{1}{2} \bar{s} \gamma^{\mu} s\left(\bar{s} \gamma_{\mu} s+\sum_{a} \bar{v}_{a} \gamma_{\mu} v_{a}\right)
\end{aligned}
$$

Finally, putting together eqs.(4.7b,d),(4.8) and (4.11), we arrive at the following expression for the continuum action:

$$
\begin{aligned}
I & =\int d^{2} \tau\left(P_{a}^{\mu} \partial_{\mu} X_{a}+c_{1} P_{a}^{\mu} P_{a, \mu}+i \bar{s} \gamma^{\mu} \partial_{\mu} s+i \bar{v}_{a} \gamma^{\mu} \partial_{\mu} v_{a}\right. \\
& +c_{2} \bar{s} \gamma^{\mu} s \bar{s} \gamma_{\mu} s+c_{3} \bar{s} \gamma^{\mu} s \bar{v}_{a} \gamma_{\mu} v_{a}+c_{4} \bar{v}_{a} \gamma^{\mu} v_{a} \bar{v}_{b} \gamma_{\mu} v_{b} \\
& \left.+c_{5} \bar{s} \gamma^{\mu} v_{a} P_{a, \mu}+c_{6} \bar{v}_{a} \gamma^{\mu} s P_{a, \mu}+c_{7} \bar{s} \gamma^{\mu} v_{a} \bar{v}_{a} \gamma_{\mu} s\right),
\end{aligned}
$$

where the summation convention for repeated indices is used, and the partials are with respect to the variable $\tau$. The constants "c" are given by

$$
c_{1}=c_{4}=c_{7}=0, c_{2}=c_{3}=\frac{1}{2} i \Lambda_{1}
$$




$$
c_{5}=-\frac{\Lambda_{1}}{2 D^{1 / 2}(2 D-2)}, c_{6}=-\frac{\Lambda_{1}(2 D-1)}{2 D^{1 / 2}(2 D-2)} .
$$

We have written eq.(4.12a) deliberately with arbitrary constants c; written in this fashion, it is the most general local action invariant under the symmetries listed after eq.(4.6). In the naive continuum limit, these constants have the values given in eq. $(4.12 \mathrm{~b})$. However, the naive continuum limit is clearly not the final answer, since under the flow of the renormalization group, these constants will be driven from their initial values given by $(4.12 \mathrm{~b})$ to some fixed point corresponding to different values for these constants. If this fixed point represents pure QCD, it is natural to expect it to have the following properties: a)It should be conformally invariant. Otherwise, renormalization would introduce a new scale in addition to the slope parameter which multiplies the action and which we have set equal to one. Since we know that pure QCD has only a single scale parameter, conformal invariance has to be an essential feature of the world sheet field theory.

b) The constant $c_{1}$ and at least one of the constants $c_{5}$ or $c_{6}$ should be different from zero at the fixed point. The non-vanishing of $c_{1}$ is necessary to have asymptotically linear Regge trajectories. With $c_{1}$ not zero, we can integrate over $\mathrm{P}$ and recover the standard quadratic term in $\mathrm{X}$. On the other hand, if both $c_{5}$ and $c_{6}$ vanished, the fermionic and the bosonic sectors of (4.12a) would decouple from each other, and the bosonic sector by itself would simply reproduce the standard string model with purely linear trajectories.( Here the labels bosonic and fermionic refer to the world sheet variables). Since we already know that the standard string model does not describe QCD, it follows that either $c_{5}$ or $c_{6}$ should be different from zero. If this is indeed the case, the resulting trajectories will be in general curved because of the coupling between $\mathrm{P}$ and the bilinears in fermions. As explained in the introduction, one expects QCD trajectories to be curved, and so this is a step in the right direction.

c) The action given by (4.12) does not look hermitian.Some of this apparent non-hermiticity can be eliminated by redefinitions of fields. On the other hand, an imaginary value for $c_{2}$, as given by eq. (4.12b), is a genuine violation of hermiticity. However, this is only the initial value of $c_{2}$ and not its value at the fixed point. Since the model should be unitary, we expect $c_{2}$ to become real at the fixed point. A related question of unitarity is whether the ghosts due to 
the Minkowski metric in target space decouple. We have nothing to say about this important problem in this paper.In any case, it is clear that to have any chance of a viable theory, the generalized Thirring model (4.12a) must have a fixed point with the properties listed above. To investigate the fixed points of this model is therefore a task of great importance for the future.

We close this section with a discussion of the continuum limit of the constraints (3.8), which supplement the action of (4.12). We remind the reader that these constraints are necessary to eliminate the spurious sectors present in the model.They could also play an important role in making the theory unitary by eliminating states with negative norm. In order to write them in covariant form, it is convenient to introduce the following vector current:

$$
J^{\mu} \equiv \bar{s} \gamma^{\mu} s+\sum_{a} \bar{v}_{a} \gamma^{\mu} v_{a}
$$

whose light cone components turn out to be the fermion densities of eq.(3.7):

$$
\begin{aligned}
& N^{(1)} \rightarrow J^{(-)}=\frac{1}{2}\left(J^{0}-J^{1}\right), \\
& N^{(2)} \rightarrow J^{(+)}=\frac{1}{2}\left(J^{0}+J^{1}\right) .
\end{aligned}
$$

The continuum analogues of eqns.(3.8a,b) are then imposed on $j^{(\mp)}$ at fixed initial values of $\sigma_{1}=\tau^{(+)}=\frac{1}{2}\left(\tau^{0}+\tau^{1}\right)$ and $\sigma_{2}=\tau^{(-)}=\frac{1}{2}\left(\tau^{0}-\tau^{1}\right)$ respectively. For these conditions to be preserved along a trajectory, J should be chirally conserved:

$$
\partial_{\mu} J^{\mu}=0, \varepsilon^{\mu \nu} \partial_{\mu} J_{\nu}=0,
$$

or, in terms of the chiral components,

$$
\partial_{+} J^{(-)}=0, \partial_{-} J^{(+)}=0
$$

These are then the continuum version of eqn.(3.7). The remaining problem is how to impose the analogues of $(3.8 \mathrm{a}, \mathrm{b})$ on $\mathrm{J}$. In the quantized theory, $\mathrm{J}$ is an operator, and it is well known that imposing conditions such as eq.(3.8) directly on the operators leads to inconsistencies. Instead, one has to impose these conditions on the physically acceptable states, using only the positive and zero modes of J. If $\tau^{( \pm)}$are compactified on an interval of length $2 \pi$, we can define 
the Fourier modes of J by

$$
J^{( \pm)}(\tau)=\sum_{n} \exp \left(i n \tau^{( \pm)}\right) J_{(n)}^{( \pm)}
$$

and write down the conditions that a physical state $\mid>$ must satisfy:

$$
J_{(n)}^{(+)}\left|>=0, J_{(n)}^{(-)}\right|>=0,
$$

for $n \geq 1$ and,

$$
J_{(0)}^{(+)}|>=|>, J_{(0)}^{(-)}|>=|>,
$$

for $n=0$.

The conservation laws given by eq.(4.14) are the consequence of the invariance of the action (4.12a) under both vector and axial $\mathrm{U}(1)$ transformations. Under vector $\mathrm{U}(1)$, the fields transform as

$$
\begin{gathered}
s \rightarrow \exp \left(i \delta_{v}\right) s, \bar{s} \rightarrow \bar{s} \exp \left(-i \delta_{V}\right), \\
v_{a} \rightarrow \exp \left(i \delta_{v}\right) v_{a}, \bar{v}_{a} \rightarrow \bar{v}_{a} \exp \left(-i \delta_{V}\right),
\end{gathered}
$$

and under the axial $\mathrm{U}(1)$, the transformation is

$$
\begin{gathered}
s \rightarrow \exp \left(i \gamma_{3} \delta_{A}\right) s, \bar{s} \rightarrow \bar{s} \exp \left(i \gamma_{3} \delta_{A}\right), \\
v_{a} \rightarrow \exp \left(i \gamma_{3} \delta_{A}\right) v_{A}, \bar{v}_{a} \rightarrow \bar{v}_{a} \exp \left(i \gamma_{3} \delta_{A}\right),
\end{gathered}
$$

where $\gamma_{3}=i \gamma^{0} \gamma^{1} . J^{\mu}$ and its dual are then the conserved currents resulting from these symmetries. We should therefore add these to the list of symmetries of the action listed after eq.(4.6). However, these symmetries are not automatic, since it is well known that axial transformations of the type given by eq.(4.17b) are in general anomalous. Such an anomaly would destroy the chiral conservation laws and would prevent us from imposing the constraints given by (4.16) consistently. To see how this anomaly comes about, we define a fermionic supermultiplet $\mathrm{Z}$ built out $D+1$ doublets s and $v_{a}$ :

$$
Z \equiv\left(\begin{array}{c}
s \\
v_{a}
\end{array}\right),
$$

and using auxilliary fields $W_{i, \mu}$, we rewrite eq.(4.12a) as

$$
\begin{aligned}
I & =\int d^{2} \tau\left(P_{a}^{\mu} \partial_{\mu} X_{a}+c_{1} P_{a}^{\mu} P_{a, \mu}+c_{5} \bar{s} \gamma^{\mu} v_{a} P_{a, \mu}+c_{6} \bar{v}_{a} \gamma^{\mu} s P_{a, \mu}\right. \\
& \left.+i \bar{z} \gamma^{\mu} \partial_{\mu} z+\sum_{i}\left(\bar{z} K_{i} \gamma^{\mu} z W_{i, \mu}+W_{i}^{\mu} W_{i, \mu}\right)\right),
\end{aligned}
$$


where $K_{i}$ are suitable $D+1 \otimes D+1$ matrices so that upon integrating over the W's, (4.12a) is reproduced. For fixed W, there is an axial U(1) anomaly proportional to the trace of the field strength built out of $\mathrm{W}$. The condition for the absence of this anomaly is that

$$
\operatorname{tr}\left(K_{i}\right)=0
$$

for all "i".At the fixed point, this implies the following two equations between the coupling constants that appear in eq.(4.12a):

$$
c_{2}=D^{2} c_{4}, c_{3}=-2 D c_{4}
$$

Clearly, the argument that led to $(4.19 \mathrm{~b})$ is only semiclassical and may be modified by higher quantum corrections. We went through this exercise merely to demonstrate that chiral $\mathrm{U}(1)$ invariance imposes further conditions on the parameters of the model.

\section{Conclusions}

The main result of this paper is the introduction of a new string model. This new model was derived by restricting the world sheet to surfaces traced by non-backtracking paths on a lattice and then taking the continuum limit. It was argued that such a restriction was the necessary minimum condition for the string theory to represent a non-abelian gauge theory in the large $\mathrm{N}$ limit. To implement this restriction on the allowed paths, fermionic variables on the world sheet were introduced, and it was shown that the world sheet action of the resulting string theory is the generalized Thirring model of eq.(4.12a). This action has to be supplemented by the conditions on admissible states given by eqs. $(4.16 \mathrm{a}, \mathrm{b})$. It was also argued that the coupling constants of the model should correspond to a fixed point, and this fixed point should satisfy a number of conditions, such as conformal invariance, in order to make connection with QCD.

Much remains to be done to find out whether the model is viable. First, one has to establish the connection between the latticized version of the string model and QCD on the lattice(10), and then, to see whether the continuum string makes sense, one has to find out whether the generalized Thirring model(4.12a) 
has a fixed point with the required properties. Hopefully, the work presented here will stimulate research along these directions.

\section{Acknowledgement}

I would like to thank Elias Kiritsis for a useful conversation. 


\section{References}

1. 1. G.'t Hooft, Nucl.Phys. B72(1974) 461.

2. 2. See, for example, A.M.Polyakov, Nucl.Phys. B268 (1986) 406.

3. 3. J.Polchinski, "Strings and QCD?", Univ.of Texas preprint, (June 1992), Phys.Rev.Letters 68 (1992) 1267.

4. 4. For some very recent work on large $\mathrm{N}$ limit of QCD, see V.A.Kazakov and A.A.Migdal, "Induced QCD at large N", Princeton preprint PUPT1322,(June 1992), Yu.Makeenko, "Large N reduction, Master Field and Loop Equations in Kazakov-Migdal Model", ITER-YM-6-92(August 1992), D.Gross, "Some Remarks about Induced QCD",Princeton Preprint, PUPT1335(August 1992).

5. 5. G.'t Hooft, Nucl.Phys. B75 (1974) 461.

6. 6. T.Eguchi, Phys.Letters B87 (1679) 91, D.Foerster,Phys. Letters B87 (1979) 87, D.Weingarten, Phys.Letters B87 (1979) 79.

7. 7. Yu.Makeenko and A.A.Migdal, Phys.Letters B88 (1979) 135.

8. 8. V.A.Kazakov, Phys.Letters B128 (1983) 316, V.I.Kostov, Phys.Letters B138 (1984) 191.

9. 9. S.Mandelstam, Phys.Rev. D19 (1979) 2391.

10. 10. K.Bardakci, work in progress.

11. 11. R.Dashen and Y.Frishman, Phys.Rev. D11 (1975) 2781.

12. 12. K.Bardakci, Nucl.Phys. B238 (1984) 621.

13. 13. For a review of Brownian motion and random walk problems, see the article by E.W.Montroll and B.J.West in "Studies in Statistical Mechanics and Fluctuation Phenomena", E.W.Montroll and J.L.Lebowitz editors, North Holland, Amsterdam (1979).

14. 14. A.M.Polyakov, Phys.Letters B103 (1981) 207. 